Nasrin Javanmardi

Parviz Ghadimi

Sasan Tavakoli

http://dx.doi.org/10.21278/brod69109

\title{
PROBING INTO THE EFFECTS OF CAVITATION ON HYDRODYNAMIC CHARACTERISTICS OF SURFACE PIERCING PROPELLERS THROUGH NUMERICAL MODELING OF OBLIQUE WATER ENTRY OF A THIN WEDGE
}

\author{
UDC 629.5.016.7:629.5.035.5:519.6
}

Original scientific paper

\begin{abstract}
Summary
The current paper investigates flow around a blade section of a surface piercing hydrofoil. To this end, a thin wedge section is numerically modelled through an oblique water entry. The flow is numerically studied using a multiphase approach. The proposed numerical approach is validated in two steps. First, pressure and free surface around a wedge entering water are simulated and compared against previously published analytical results. Subsequently, cavitation phenomenon around a submerged supercavitating hydrofoil is modelled and analyzed. It is observed that cavity length, pressure, and lift force are accurately predicted. Subsequently, the main problem has been studied for two different cavitation numbers for a range of advanced ratios equivalent to fully, transition and partially ventilated conditions in order to investigate the effect of ambient pressure on hydrodynamics of the water entry of the foil. The numerical findings reveal that, when the cavitation number decreases, the start of transition mode is postponed and this mode is expanded for the larger range of velocity ratios. This implies that fully ventilated velocity ratio modes are expanded, too. However, in the transition mode, the cavitation number plays an essential role and may lead to a decrease in the pressure difference across the surface piercing hydrofoil which yields a decrease in the resultant force.
\end{abstract}

Key words: $\quad$ Surface piercing propeller; oblique water entry; thin wedge; Cavitation number; Numerical modelling;

\section{Introduction}

Surface piercing propellers (SPP) have various applications for different high-speed marine crafts including planing boats and surface effect ships (SES). When the blade of such a propeller enters the water, ventilated air is generated by gas formation. In addition, for many applications of these propellers where the pressure drastically drops, the possibility of this blade undergoing cavitation phenomenon increases. Consequently, a combination of water, 
Probing into the effects of cavitation on hydrodynamic characteristics of surface piercing propellers through numerical modeling of oblique water entry of a thin wedge

vapor, and air is generated near the blade, as it enters the water and the physics of the flow becomes quite complicated. From an engineering point of view, it is imperative to quantify the thrust force and the generated moment in the presence of cavitation which can be quite beneficial in the design process.

Shiba [1] is considered a pioneer in investigating the ventilation and cavitation mechanism of surface piercing objects. To ascertain the effective parameters on these phenomena, he conducted different experiments on the surface piercing (SP) hydrofoils. Based on the obtained experimental results, he concluded that ventilation occurs when the pressure in downstream of the surface piercing object drops below the ambient pressure minus the pressure associated with the surface tension. The feasibility of using supercavitating sections for the propellers in partially submerged conditions was first investigated by Hadler [2] and Hacker [3]. They conducted experimental measurements on the SP hydrofoils and SP propellers. Three main flow regions of fully ventilated, transition, and partially ventilated were initially introduced by them. Kruppa [4] experimentally modeled surface piercing propellers and reported some results on the effects of Froude numbers and Reynolds numbers on scaling. Meanwhile, Brandt [5] used some of his experimental data and concluded that the point at which the transition mode starts depends on the cavitation and Froude numbers. Olofsson [6] measured the forces and torque of a SPP model 821-b. He reported these parameters during one cycle. By conducting a series of experiments, he found that during water entry process of the SP blades, the decrease in cavitaion number may lead to an increase in the forces and moments, which was declared as quite unusual by him. However, experimental research by Shen [7] on 2D and 3D hydrofoils showed both increase and decrease in the lift force with the appearance of cavitation.

Numerical methods have also been utilized for investigating the submerged and surface piercing propellers. Furuya [8] presented the results of numerical simulation of the flow around a surface piercing propeller. His results were restricted to fully ventilated condition. Wang [9], on the other hand, used a lifting surface method to numerically model these propellers. Later, Kudo [10] used VLM method to model the SPPs. Also, Young and Kinnas [11] used surface panel method and BEM and analyzed flow around the surface piercing hydrofoils and propellers and reported the existence of cavitation phenomenon around the SPP blades. Through the same approach, Young and Savander [12] conducted hydroelastic analyses of large size SPPs and examined the effects of diameters on their hydroelasticity. Ghassabzadeh et al [13] have also applied this approach to evaluate hydroelastic behavior of the submerged propeller. Similar to the SPPs characteristics, operation close to the water free surface or percents of cavitation sheets could also affect the common marine propeller performances. The effects of these phenomena have been numerically studied by Ekinci et al [14], Bagheri et al [15], Kinaci et al. [16] and Samir et al [17].

Investigation of flow around surface piercing propellers can alternatively be accomplished through an oblique wedge water entry. Theoretical research of Yim [18] may be considered as one example of such approach. He presented simulation of water entry of thin wedges which were infinite and reported that such a solution may be useful for analyzing flow around surface piercing propellers. Wang [19] further developed these solutions in order to consider the fully ventilation mode of the flow for a hydrofoil. Later, Cox [20] experimentally studied water entry problem of thin wedges where the solution by Wang [19] was modified in such a 
way that gravity effects is taken into consideration. Judge et al. [21] presented a numerical simulation for the potential flow around a wedge entering water with an oblique speed. They presented new boundary conditions for solving this problem. Afterward, Faltinsen and Semenov [22] presented a nonlinear theoretical simulation of the flow of an oblique water entry, where they provided a specific conformal mapping for solving of the problem. On the other hand, $\mathrm{Xu}$ et al. [23] presented numerical simulation of the oblique water entry of asymmetric wedges. Xu et al. [24] later extended their simulation in such a way that freefall of the wedge was considered. Recently, there has been good progress in numerical modeling of the flow related to water entry problems. Application of analytical method by Ghadimi et al. [25], URANS method by Ghadimi et al. [26-28], as well as SPH simulations by Farsi and Ghadimi [29-31] and Feizi Chekab et al. [32] in simulations of water entry of different objects have suggested that numerical solution of the viscous flow may be an appropriate choice for analyzing flow around wedges.

The main objective of the current paper is to offer further contribution to the understanding of cavitation phenomenon in surface piercing propellers through the usage of computational fluid dynamics and by investigating flow around a wedge. In this framework, different parametric analyses are conducted on a particular 2D blade section for two different cavitation numbers and various advanced ratios ranging from 0.23 to 0.64 . Three different operating conditions including fully ventilated, transition, and partially cavitated are also considered. A purposive validation is presented through comparison in two steps. Finite volume based software ANSYS-CFX is employed as solver. Ultimately, the main results of the paper, which include the free surface, pressure, and forces acting on the wedge, are presented and it is illustrated how these factors are affected, as the impact velocity and cavitation number changes.

\section{Problem Definition}

\subsection{Geometry of the problem}

A surface piercing hydrofoil is considered to penetrate the water with vertical velocity of $\mathrm{V}$, which may also have been influenced by an inlet velocity of $\mathrm{U}$. This section is similar to a wedge whose deadrise angle is denoted by $\beta$. The ambient pressure is denoted by $\mathrm{P}_{\mathrm{atm}}$ which can be varied. Variation of this pressure may help initiate different cavitation numbers. During this type of water entry, three different conditions can be observed. These conditions may also occur for a surface piercing propeller. The first condition is called a fully ventilated mode in which water surface separates from the wedge apex in the leading edge and consequently, the air is sucked into the water (Fig. 1a). In this condition, the fluid behavior is perfectly steady and the hydrodynamic pressure acts only on the pressure side. For the constant inlet velocity $\mathrm{U}$, if the impact velocity $\mathrm{V}$ decreases, a different condition appears which is called transition mode (Fig. 1b). In this condition, the wall of air cavity impacts the foil body and as a result, the cavity is disconnected from the atmosphere. Meanwhile, the pressure does not display a steady behavior and is highly affected by the speed ratios. As the impact velocity continues to decrease, a third condition called partially cavitation mode occurs in which the cavitation sheet begins from the leading edge and can remain on the suction side. As a result, no ventilation pattern is observed on the free surface (Fig. 1c). 


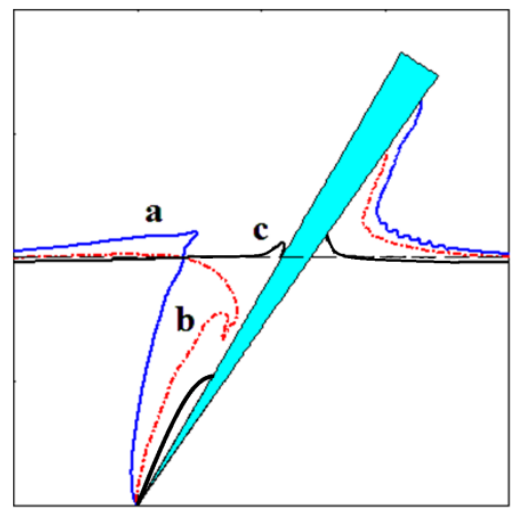

Fig. 1. Problem definition

\subsection{Application of the considered problem for the surface piercing propellers}

In Fig. 2. Through a close scrutiny of the blade of this type of a propeller, its section, and the presented velocities attacking the section (Fig.2), one may conclude that a threedimensional problem can be changed to a $2 \mathrm{D}$ water entry problem with an oblique speed, which is the exact replica of the problem introduced in the previous sub-section.

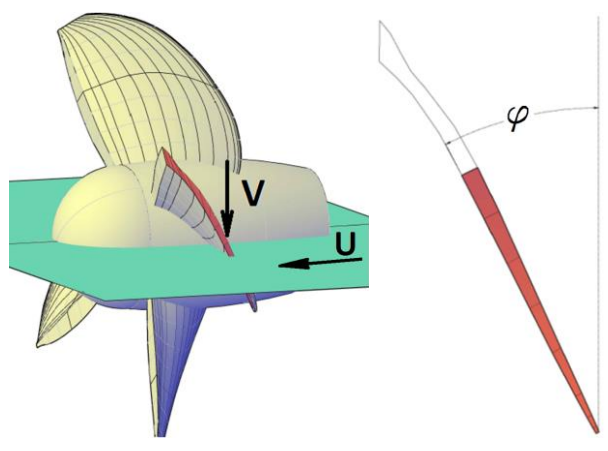

Fig. 2. SPP geometry and the hydrofoil section.

The propeller has a diameter $D$ and is considered to rotate with an angular speed of $n$. The advanced ratio of the propeller is determined by

$J=\frac{U}{n D}$

The dominant thrust and torque on the rotating propeller are found to be in the range of $0.5 \mathrm{r}$ to $0.7 \mathrm{r}$. Therefore, a better approach which can bring about meaningful results may be the consideration of a section between these two radii. Accordingly, the current paper focuses on the section $0.55 \mathrm{r}$ from the hub. The impact velocity of this section can be computed by

$V=0.55 \pi n D$

The ratio of the inlet velocity to the impact velocity is defined by $\mathrm{U} / \mathrm{V}$ which is found by 
$\varepsilon=U / V=\frac{J}{0.55 \pi}$.

The Froude number associated with the propeller is also defined by

$F r=\frac{U}{\sqrt{g D}}$. by

In most of the research papers conducted on propellers, the cavitation number is defined

$\sigma_{n}=\frac{\left(P_{0}-P_{v}\right)}{0.5 \rho_{W} U^{2}}$,

where $P_{v}$ is the vapor pressure of the water and $\mathrm{P}_{0}$ is the hydrostatic pressure at the propeller hub and since the hub of SPP is not submerged, $P_{0}=P_{a t m}$ condition is assumed. The pressure acting on the blade section is normalized using the relation

$C_{P}=\frac{\left(P-P_{0}\right)}{0.5 \rho_{W} U^{2}}$.

The force acting on the 2D wedge, which is obtained by integrating the pressure over the blade section, is non-dimensionalized using the relation

$K_{F}=\frac{F}{0.5 \rho_{w} V^{2} h}=\frac{F}{0.5 \rho_{w} V^{3} t}$,

where, $h$ is the foil submergence at time $t$ which is equal to V.t. Meanwhile, the efficiency of the water entry process of a wedge, similar to the definition of propeller efficiency, is computed by

$\eta_{\text {Wedge }}=\frac{F_{x} U}{F_{y} V}$,

where $F_{x}$ and $F_{y}$ are the horizontal and vertical forces acting on the wedge, respectively.

\subsection{Governing equations of the fluid}

Since the present study deals with three different phases of vapor, water, and air, the homogeneous mixture model is used to calculate the mixture pressure and velocity. Accordingly, based on this model, the continuity and momentum URANS equations are implemented as

$$
\frac{\partial\left(\rho_{m}\right)}{\partial t}+\nabla \cdot\left(\rho_{m} \vec{V}\right)=0
$$




$$
\frac{\partial\left(\rho_{m} \vec{V}\right)}{\partial t}+\nabla \cdot\left(\rho_{m} \overrightarrow{V V}\right)=\rho_{m} g-\nabla P+\nabla \cdot\left[\mu_{m}\left(\nabla \vec{V}+\vec{W}^{T}\right)-\frac{2}{3} \nabla \overrightarrow{V I}\right],
$$

where $I$ is the unit tensor, while $\rho_{\mathrm{m}}$ and $\mu_{m}$ are the mixture density and viscosity, respectively, which can be computed by

$$
\begin{aligned}
& \rho_{m}=\sum \alpha_{n} \rho_{n}=\alpha_{a} \rho_{a}+\alpha_{v} \rho_{v}+\left(1-\alpha_{a}-\alpha_{v}\right) \rho_{w}, \\
& \mu_{m}=\sum \alpha_{n} \mu_{n}=\alpha_{a} \mu_{a}+\alpha_{v} \mu_{v}+\left(1-\alpha_{a}-\alpha_{v}\right) \mu_{w} .
\end{aligned}
$$

In the above equation, subscripts $a, v$, and $w$ denote air, vapor, and water fluid, respectively. $\alpha_{n}$ is the volume fraction of the $n$-th fluid in a cell, and it is known that

$$
\sum \alpha_{n}=1
$$

The mass continuity equation for the existing mixture can be written in the form of three different equations as in

$$
\begin{aligned}
& \frac{\partial\left(\alpha_{a} \rho_{a}\right)}{\partial t}+\nabla \cdot\left(\alpha_{a} \rho_{a} \vec{V}\right)=0, \\
& \frac{\partial\left(\alpha_{W}\right)}{\partial t}+\nabla \cdot\left(\alpha_{W} \vec{V}\right)=-\frac{\dot{m}}{\rho_{W}}, \\
& \frac{\partial\left(\alpha_{V} \rho_{V}\right)}{\partial t}+\nabla \cdot\left(\alpha_{V} \rho_{V} \vec{V}\right)=\dot{m},
\end{aligned}
$$

$\dot{m}$ is the mass transfer rate that is only observed between the water vapor phases. By using the proposed evaporation and condensation model of Zwart [33], it can be written that

$$
\begin{aligned}
& \dot{m}=\dot{m}_{e}-\dot{m}_{c}, \\
& \dot{m}_{e}=C_{e} \frac{3\left(1-\alpha_{V}\right) \alpha_{n u c} \rho_{V}}{R_{b}} \sqrt{2\left(P_{V}-P_{o}\right) / 3 \rho_{w}} \operatorname{sgn}\left(P_{V}-P_{o}\right), \\
& \dot{m}_{C}=C_{C} \frac{3 \alpha_{V} \rho_{V}}{R_{b}} \sqrt{2\left(P_{O}-P_{V}\right) / 3 \rho_{w}} \operatorname{sgn}\left(P_{O}-P_{V}\right),
\end{aligned}
$$



characteristics of surface piercing propellers through numerical modeling of oblique water entry of a thin wedge

where $C_{e}$ and $C_{c}$ are empirical values being equal to 50 and 0.01 , respectively. $R_{b}$ is the mean radius of the bubble and is assumed to be $1 \times 10^{-6} \mathrm{~m}$, as proposed by $\mathrm{Ji}$ et al. [34]. $\alpha_{V}$ is the vapor fraction which is considered to be $5 \times 10^{-4}$, as proposed by Mejri et al. [35].

\section{Problem Set Up}

For solving the problem, the displayed domain in Fig. 3 is considered. Figure 3 shows the geometry of a wedge (supercavity hydrofoil) penetrating the water with impact speed of $V$, while the water enters the domain from the left side with a speed of $U$. Initially, the wedge apex is located at the water free surface.

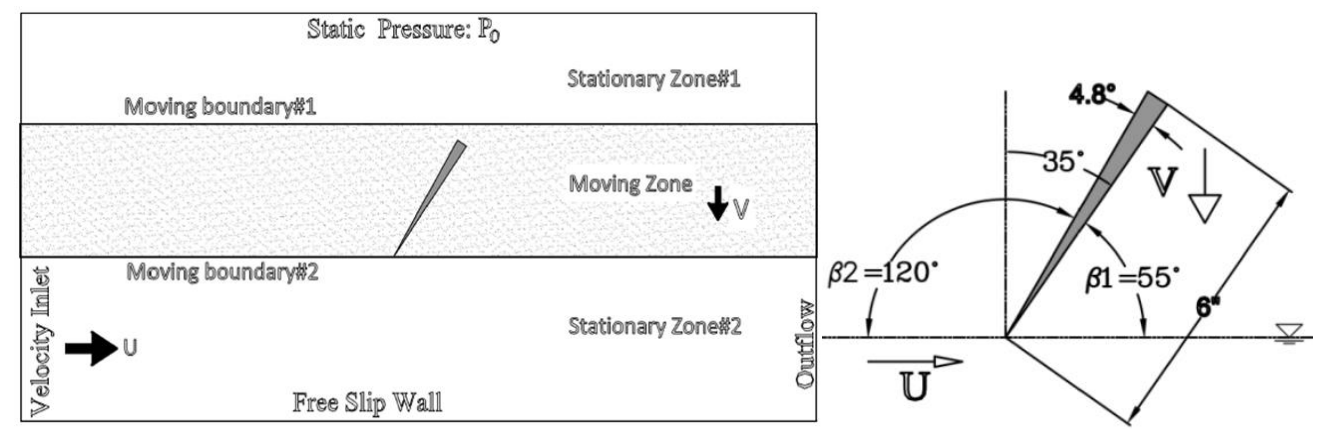

Fig. 3. The considered foil section geometry and domain of the current problem.

The computational domain is discretized using a structured mesh. The cells have a hexahedron shape. The wedge walls are discretized using boundary layer mesh approach. Three different sub-domains are taken into consideration. As evident in Fig. 3, the moving zone cells move rigidly toward the set free surface, which cause the upper and lower zone cells to be decompressed and compressed, respectively. Therefore, in order to properly mesh the stationary sub-domains, a dynamic meshing approach is adopted. As a result, 0.5 million cells are produced. The discretized domain is illustrated in Figs. 3 and 4.

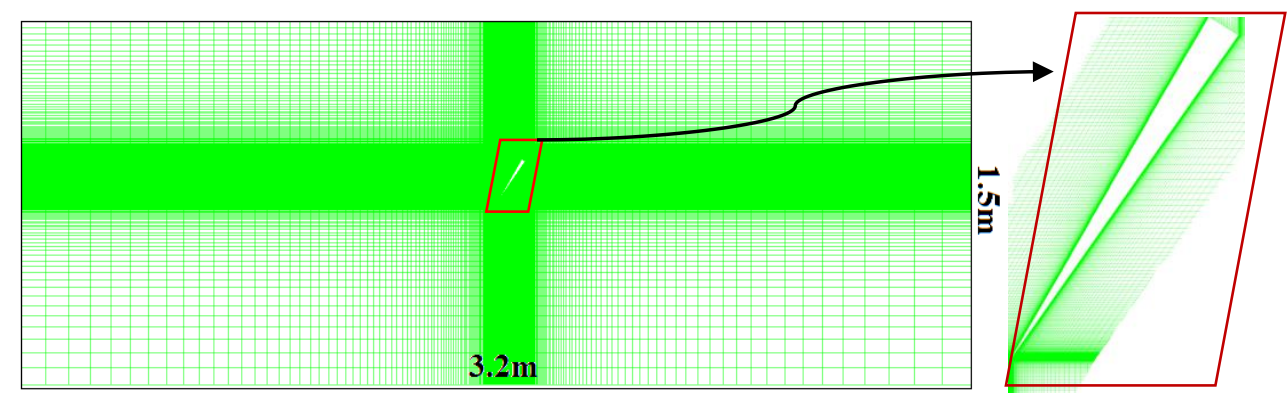

Fig. 4. The generated mesh.

\section{Validation}

The considered validation of the proposed numerical model consists of two different parts. In the first part, a wedge water entry is studied, while in the second part, cavitation around a supercavitating hydrofoil is modeled. In each of these parts, one aspect of the current simulation is validated.

\subsection{Wedge entry to a calm water tank}

Tveitnes [36] experimentally studied hydrodynamic characteristics of a series of symmetric wedges water entry. These experiments were conducted in calm water condition. The schematic of the wedge and simulated domain setup are displayed in Fig.5. 


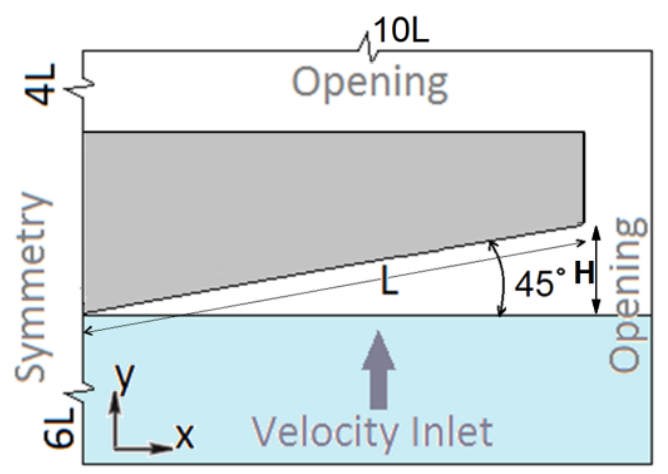

Fig. 5. The wedge domain boundary conditions and the involved geometry.

The validation case involves the water entry of a wedge whose length is $\mathrm{L}=0.3 \mathrm{~m}$ and deadrise angle is 45 degrees. The wedge impacts the calm free surface with a constant vertical speed of $\mathrm{V}=1.19 \mathrm{~m} / \mathrm{s}$. Free surface pattern of the wedge at $V . t=0.5 \mathrm{~m}$ is illustrated in Fig.6 (left) and the obtained dimensionless forces during the wedge motion are compared against the experimental results of Tveitnes [36] in Fig.6 (right).
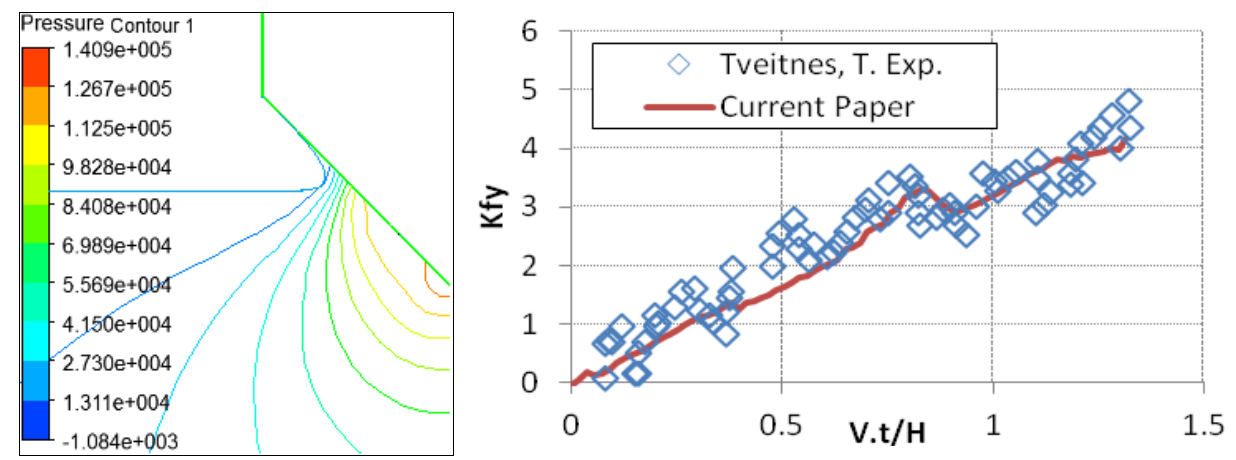

Fig. 6. The obtained free surface pattern at V.t=0.5m (left) and comparison of the obtained forces with experimental data from Tveitnes [36](right).

As evident in Fig.6 (right), there is favorable agreement between the computed force coefficients and experimental data.

\subsection{Cavitation}

Here, a supercavity foil is numerically modeled by the proposed approach and the results are compared against available data. This foil was experimentally studied by Dinh [37]. This foil is a supercavity foil which is attacked by the flow at 9 degrees. The profile of this foil is displayed in Fig. 7.

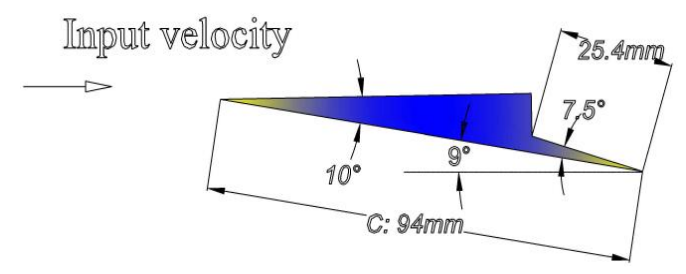

Fig. 7. Profile of the considered foil by Dinh [37].

This hydrofoil is modeled for five different cavitation numbers, cavity length, the pressure, and the acting lift on the body are computed. The computation is performed with 100,000 cells. The cavity shape is shown in Fig. 8 ( $c$ is the cord length). As evident in this figure, 

characteristics of surface piercing propellers through numerical modeling of oblique water entry of a thin wedge

throughout the current simulation, length of the cavity increases as the cavitation number decreases, which is similar to what happens in reality.

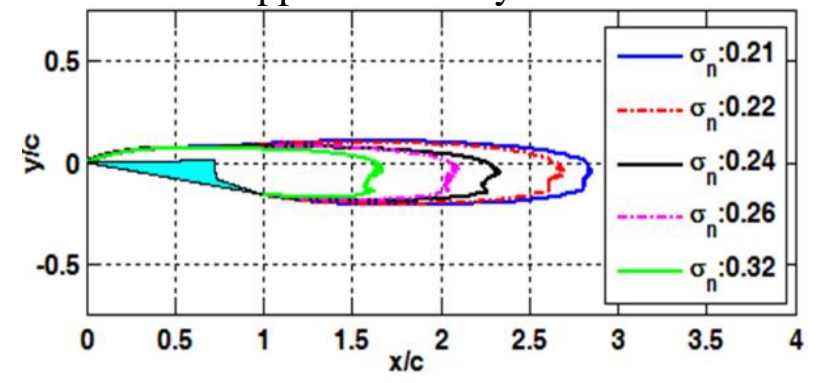

Fig. 8. Obtained cavity shapes.

The computed cavity length and the experimental data of Dinh [37] are displayed in Fig. 9. Based on the reported data, the results of the current simulation display relatively good agreement with the experimental results of Dinh [37] and there is only a slight underprediction. It should be noted that radius of hydrofoil's leading edge also has important effects on the generated cavity length. Since this value is unknown, it is set to be zero which leads to some errors. However, the RMS of the errors between the computed cavity length and that of Dinh's experiment is determined to be 0.1 which is considerably small (Table 1 ).

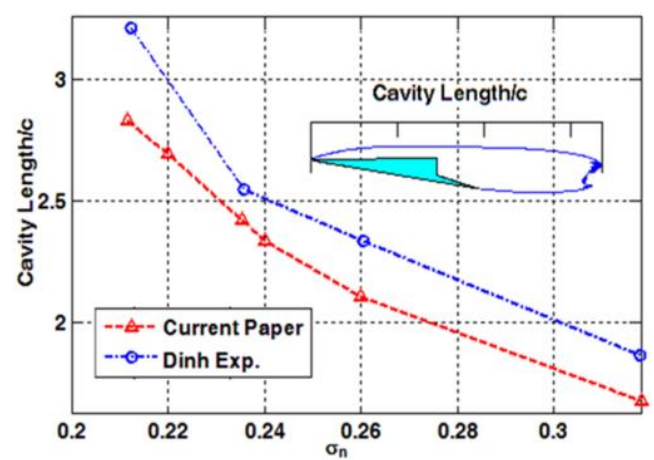

Fig. 9. Comparison of the computed cavity length with experimental data of Dinh[37].

Table 1. RMS error calculation.

\begin{tabular}{lc}
\hline$\sigma_{n}$ & Error\% \\
\hline 0.21 & $11 \%$ \\
\hline 0.236 & $8 \%$ \\
\hline 0.26 & $10 \%$ \\
\hline 0.32 & $10 \%$
\end{tabular}

The computed pressure and experimental data of Dinh [37] are displayed in Fig. 10. The results of free-streamline theory by $\mathrm{Wu}$ [38] are also presented in this figure. As evident in this figure, the prediction of the pressure distribution shows proper accuracy which confirms the effectiveness of the proposed model. Finally, the computed lift forces are illustrated in Fig. 11. In this figure, the measurements of Dinh [37] and the analytical results of Wu [38] displayed. As observed in Fig. 11, the current simulation displays proper accuracy for all the cavitation numbers. 

modeling of oblique water entry of a thin wedge

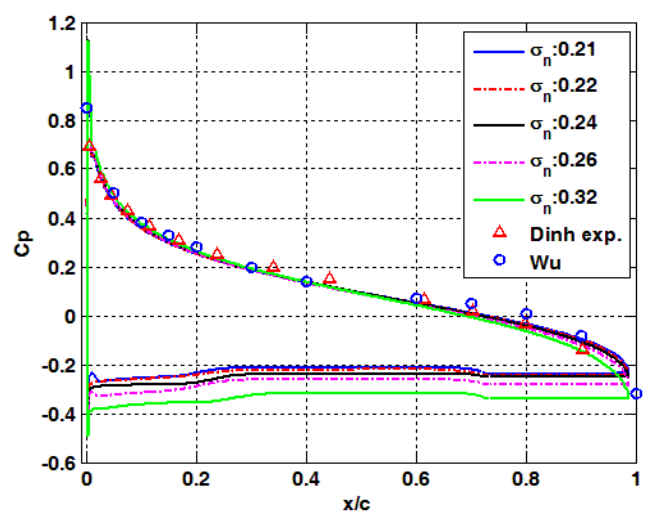

Fig. 10. Comparison of the computed cavity length with experimental data of Dinh [37].

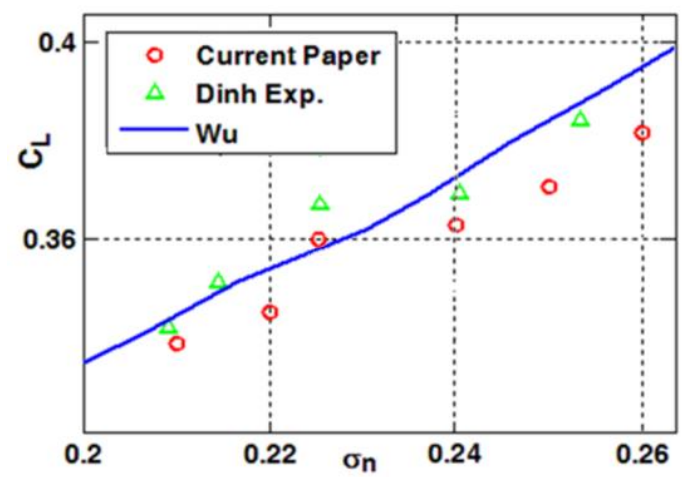

Fig. 11. Comparison of the computed lift force against experimental data of Dinh [37].

\section{Results and Discussion}

A supercavitating blade section with deadrise angle of 120.2 degrees on the left side and 55 degrees on the right side (Fig.3) is investigated for different cavitation and Froude numbers (Table 2). The corresponding results are discussed in this section. Two different ambient pressures corresponding to two cavitation numbers are considered. First, it is considered that the ambient pressure is equal to the atmospheric pressure. It is then assumed that ambient pressure is equal to $1 / 7$ of the atmospheric pressure. The later pressure is selected based on Olofsson's [6] experimental results on SPP 821-b. During his experimental investigations for the ambient pressure below the stated value, the effects of cavitation number on the characteristic curves were proved to be significant. Through such approach, the effects of cavitation on the flow pattern, pressure, and forces may be determined easier. Also, three different $U / V$ ratios ranging from 0.23 to 0.64 are taken into consideration.

Table 2. Parameter settings for the conducted analyses.

\begin{tabular}{ll}
\hline Parameter & Value \\
\hline Velocity ratio (U/V) & $0.23-0.64$ \\
Froude number & 6 \\
Cavitaion Number & $0.25,2.3$ \\
\hline
\end{tabular}

\subsection{Ventilation Pattern}

Ventilation regime on the water encountering the hydrofoil can be detected by the observed free surface profile and the recorded pressure on the foil's walls. Therefore, in this section, variation of these two parameters are computed for different $U / V$ ratios and presented in Fig.12. All the results in this sub-section are presented for the cavitation number of 2.3. As evident in Fig. 12, through an increase in U/V (or increase in the advanced ratio), the free 

characteristics of surface piercing propellers through numerical modeling of oblique water entry of a thin wedge

surface on the cavity wall tends to get closer to the back side of the wedge. However, this phenomenon displays different conditions for different velocity ratios. At lower velocity ratio (high vertical wedge velocity), i.e. $U / V=0.23$, the fluid flow detaches from the leading edge and free surface pattern corresponding to the fully ventilated regimes appears. Based on Shiba's observations [1] outlined in the literature, higher angle of attack of the hydrofoil causes higher pressure difference during the water entry and air is thus perfectly sucked into the low pressure region. The cavity peak point $\mathrm{C}$ (shown in Fig.14) is far enough from the wedge to balance the pressure in the cavity with the ambient pressure. The hydrodynamic pressure only acts on the face side of the foil and it has a steady behavior in this case (Condition I in Fig.12). Afterward, as $U / V$ increases $(U / V=0.38)$, cavity peak point $\mathrm{C}$ is adhered to the back side of the wedge and the cavity is disconnected from the ambient air. This condition was earlier defined as the transition mode (Condition II in Fig.12). When the ventilated cavity collapses, the closed vapor cavity near the leading edge remains on the back side of the wedge and partially cavitation regime occurs. Accordingly, the pressure would have a steady condition and the resultant force substantially drops (Condition III in Fig.12).
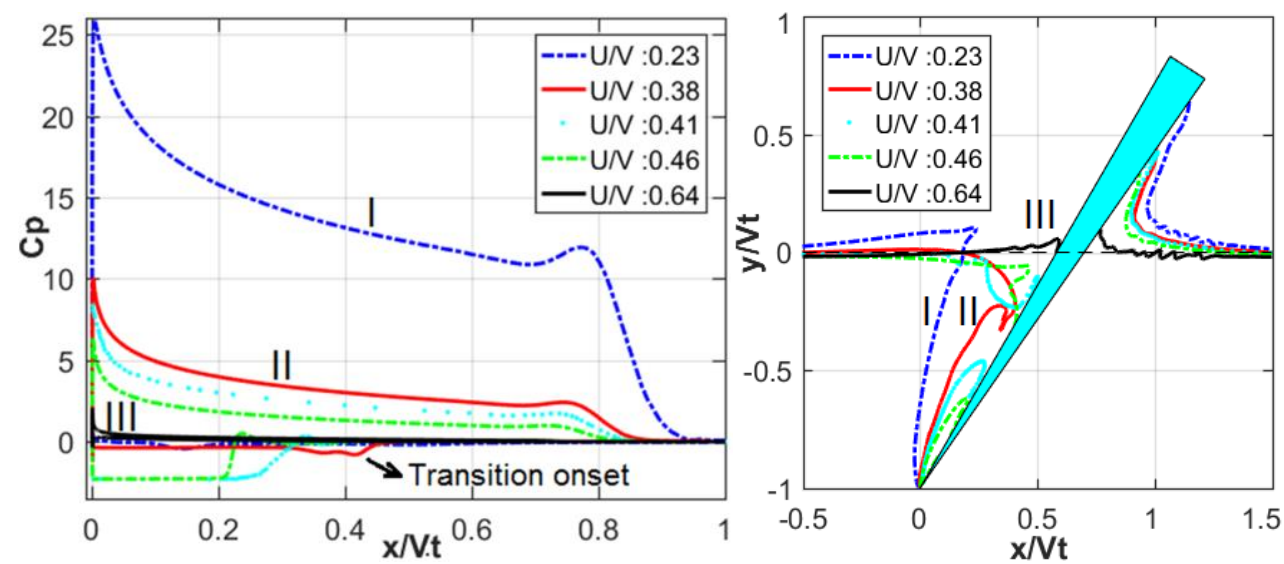

Fig. 12. Pressure distribution and water surface profile for different values of $U / V$ at $F n=6$ and $\sigma=2.3$. Vt or V.t (product of velocity and time is height) is the foil submergence at time $t$.

The horizontal and vertical forces corresponding to each value of $U / V$ are displayed in Fig. 13 which include the results in all three considered conditions or regimes and may help understand the effect of each of these conditions on the forces. As observed in Fig.13, through an increase in $U / V$ in both fully ventilated and partially cavitation conditions, dimensionless values of $F_{x}$ and $F_{y}$ are reduced. This is while the efficiency of the blade section increases. This may be attributed to the reduction of the spray height on the pressure side of the wedge and consequently the decrease in the wasted energy, as the velocity ratio increases. As a result, the efficiency should increase in both of these modes. However, there is an exception in the case of partially cavitation mode, where the impact velocity highly decreases and hence the wetted area increases considerably in such a way that the efficiency drops. Such phenomena can be seen for $U / V=0.64$. The main aspect and the most important fact which should be considered here are the resultant forces in the transition mode. In this mode, since the ventilated cavity is disconnected from the atmosphere, the pressure drops in this area (see pressure distribution and cavity profile for $U / V=0.38$ in Fig.12.II). Through this sudden drop, the difference of the pressure between the sides highly increases and the resultant force increases. 


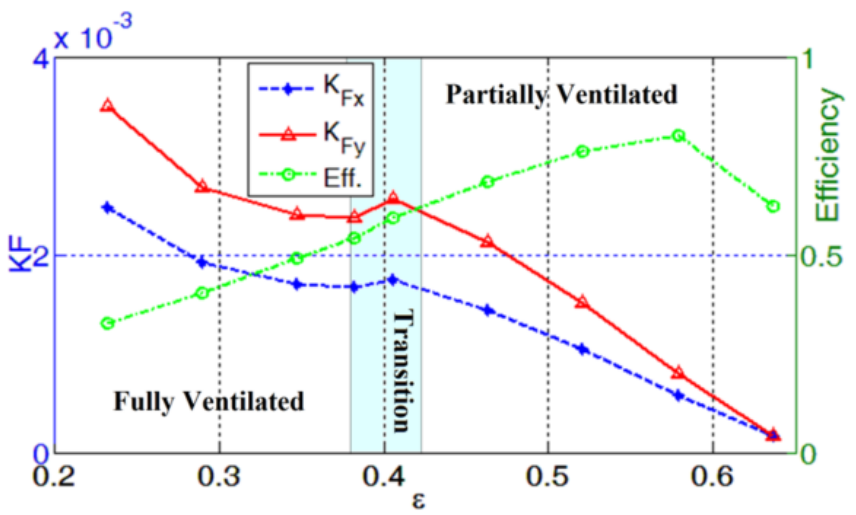

Fig. 13. Plots of $F_{x}$ and $F_{y}$ for the considered hydrofoil section at $F n=6$ and $\sigma=2.3$.

\subsection{Effects of the ambient pressure}

Fully Ventilation. A comparison is presented in Fig.14 between the pressure distribution and water surface profile of the fully ventilated condition $(U / V=0.23)$ for two cavitation numbers of $\sigma=2.3$ and 0.25 . A close scrutiny of this figure indicates that there is no significant difference between the free surface profiles.
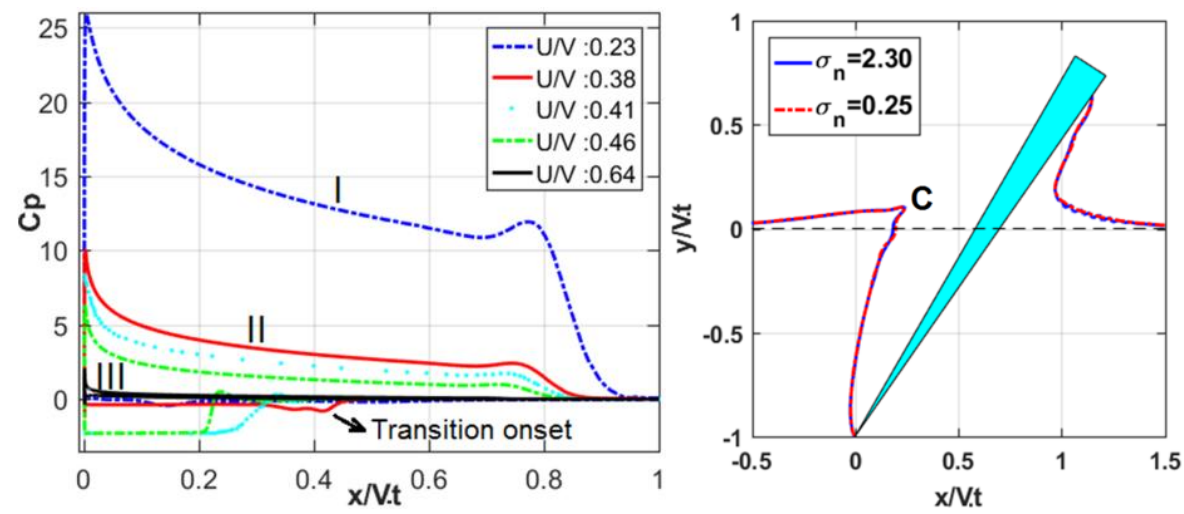

Fig. 14. Pressure distribution (left) and water surface profiles (right) in fully ventilated condition $(U / V=0.23)$ and Fn=6.0 for cavitation numbers $\sigma=0.25$ and $\sigma=2.3$.

This may be attributed to the fact that cavity pressure at both cavitation numbers is equal to the ambient pressures $\left(P_{0}: 1 \mathrm{~atm}, P_{0}{ }^{\prime}: 1 / 7 \mathrm{~atm}\right)$ and that there is enough space for the air in both cases to penetrate the ventilated area. Therefore, only pressure in the case of lower ambient pressure, drops. The pressure and water surface profile for a larger $U / V$ (i.e. $U / V$ $=0.38$ ) in the fully ventilated condition are shown in Fig.15. With a decrease in $\mathrm{V}$, the position of point $\mathrm{C}$ (shown in Fig.14) gets closer to the back side of the wedge. As a result, the air moving toward the foil's leading edge exhibits a pressure drop at point $\mathrm{C}$. At point $\mathrm{C}$ (hereby called "Critical C"), a particular region is established due to this pressure drop in which the separation of the air cavity is expedited. This phenomenon may block the path of air moving toward the leading edge faster which itself leads to a separation. If the ambient pressure is lower (e.g. $\sigma=0.25$ ), through a slight pressure decrease in the closed cavity region, the pressure may reach the vapor pressure and the cavity wall hence experiences cavitation. This cavitation causes an injection of vapor into the cavity and prevents point $\mathrm{C}$ from being further sucked down. This is why point $\mathrm{C}$ is located at an upper position for the case of lower cavitation number. 

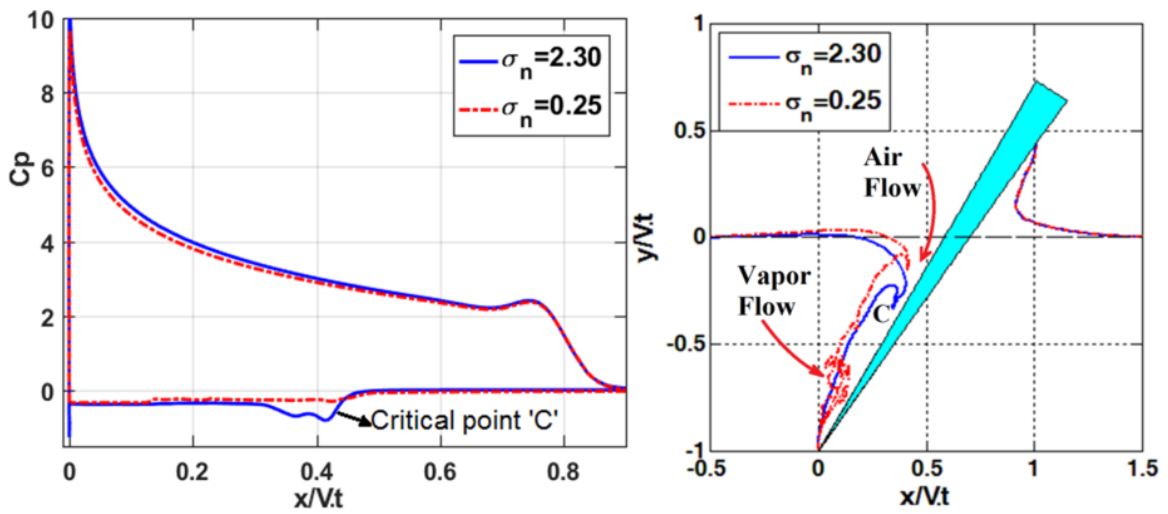

Fig. 15. Pressure distribution (left) and water surface profiles (right) in fully ventilated condition $(U / V=0.38)$ and $\mathrm{Fn}=6.0$ for cavitation numbers $\sigma=0.25$ and $\sigma=2.3$.

Transition condition: As stated and explained earlier, with an increase in $U / V$ ratio, the flow may turn into another mode called transition mode, in which air is divided into two regions. In order to explore the effects of cavitation number on the free surface and pressure in this flow mode, these two factors are computed and displayed in Fig. 16 at $U / V=0.4$ for two different cavitation numbers. As mentioned in the previous subsections, there should be a large difference between the pressures $(\Delta P)$ of both sides in this case. However, for the case of lower ambient pressure, as evident in Fig.15, the vaporization does not allow the water surface to come down and adhere to the back side of the wedge. Therefore, the transition regime signs are not yet observed. In this phenomenon, the pressure difference $\left(\Delta P^{\prime}\right)$ in the case of lower cavity number, decreases.
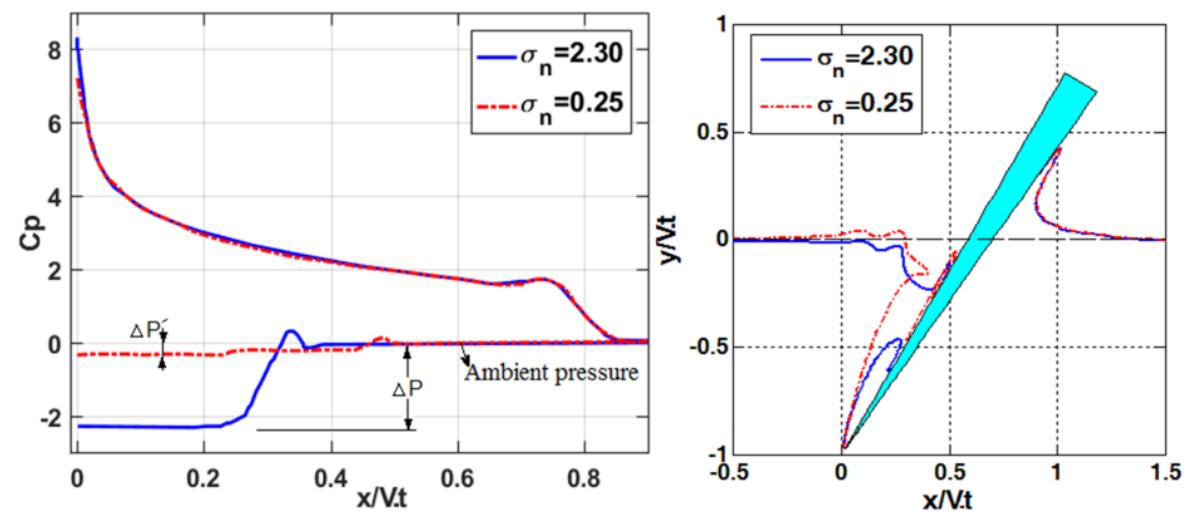

Fig. 16. Pressure distribution (left) and water surface profiles (right) for cavitation numbers $\sigma=0.25$ and $\sigma=2.3$ at $U / V=0.4, F n=6.0$

Free surface pattern and pressure distributions of both considered ambient pressures for $U / V=0.48$ are shown in Fig.17. Based on the obtained results, it may be concluded that the transition in the case of lower Cavitation number occurs with a delay, in comparison with the case of larger Cavitation number. Therefore, while the transition happens at $U / V \sim 0.38$ for the case of $\sigma=2.3$, this phenomenon is shifted for the case of $U / V \sim 0.48$ at $\sigma=0.25$. As observed in Fig.17, the free surface in the case of smaller Cavitation number experiences the transition mode. However, pressure is the important aspect of this physical phenomenon. Although the case with smaller Cavitation number exhibits transition condition, the difference between pressures of both sides is smaller for this case, in comparison with that of larger Cavitation number. 

modeling of oblique water entry of a thin wedge
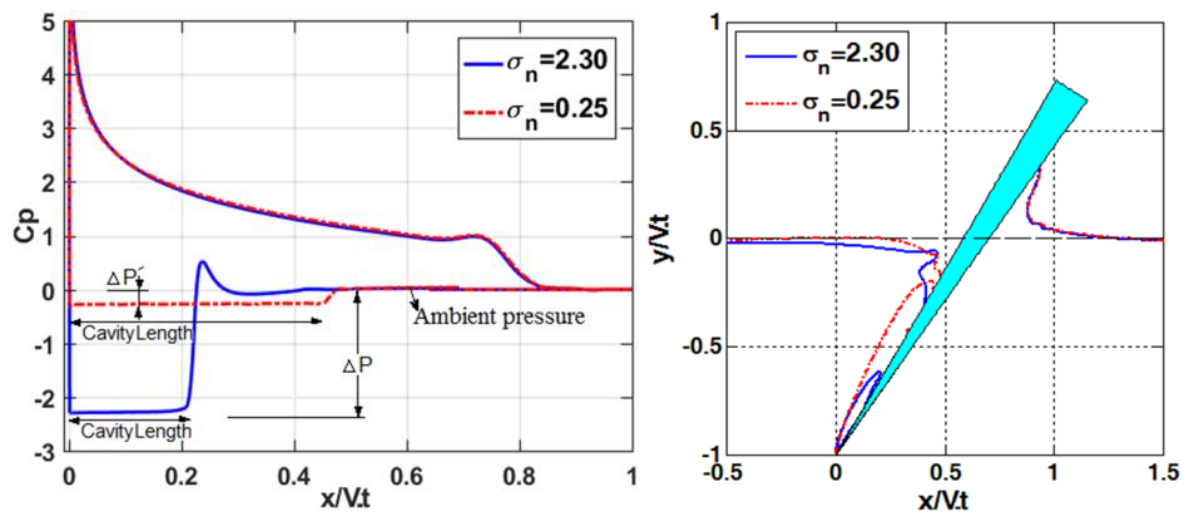

Fig. 17. Pressure distribution (left) and water surface profiles (right) at $U / V=0.46$ and $F n=6.0$ for cavitation numbers $\sigma=0.25$ and $\sigma=2.3$.

Partially cavitation condition: As the velocity ratio increases (i.e. $V$ decreases), no ventilation occurs and only cavitation sheet is observed near the leading edge, in which case partially cavitation mode occurs. The free surface and pressure distribution for $U / V=0.52$ are shown in Fig.18. Based on the free surface plots, it can be concluded that the free surface profiles for both cases are similar. According to the plots of pressure distributions, it may be claimed that pressures of the face sides for both cases are also similar and there is a downward shift, similar to the previous cases. However, on the suction side area, the generated vapor cavity in the case of lower ambient pressure, is longer than the case of higher ambient pressure.
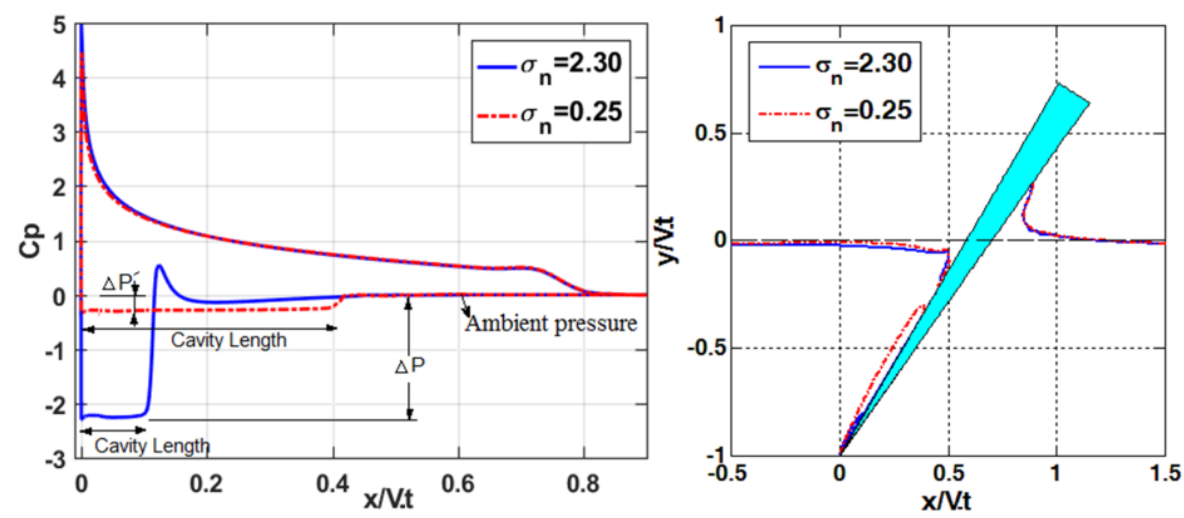

Fig. 18. Pressure distribution (left) and water surface profiles (right) in $U / V=0.52$ and $F n=6.0$ for cavitation numbers $\sigma=0.25$ and $\sigma=2.3$.

Figure 19 illustrates the results for another $U / V$ ratio in a different partially cavitation case. The considered ratio corresponding to these results is $U / V=0.64$. In this situation, it is also observed that free surface profile and pressure distribution are similar in both cases, except for the fact that pressure is shifted downward. Similarly, equivalent forces are expected to be produced in both cases which will be presented in the next subsection. Therefore, one may conclude that when $U / V$ ratio increases enough to avoid cavitation occurrence, the cavitation number would not be a significant contributor. 

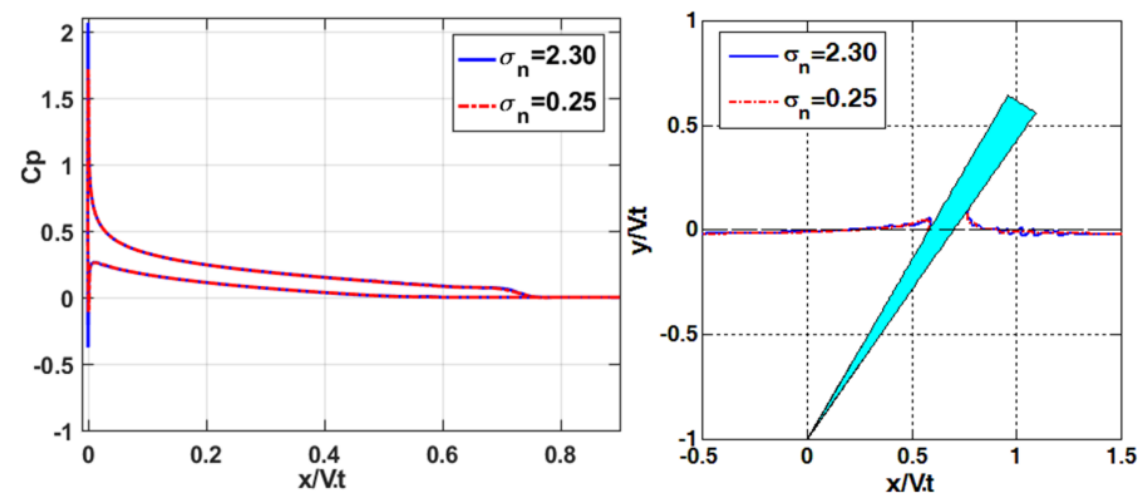

Fig. 19. Pressure distribution (left) and water surface profiles (right) at $U / V=0.64$ and $F n=6.0$ for cavitation numbers $\sigma=0.25$ and $\sigma=2.3$.

Effects of cavitation number on the performance curves: The computed forces as well as the efficiency are displayed in Fig. 20, as a function of $U / V$. All the run conditions are related to Froude number of $F n=6$ and $U / V$ ratios ranging from 0.23 to 0.64 . In the previous subsections, the pressure distribution and the effects of cavitation number were discussed. The results in Fig. 20 may help derive a general conclusion regarding the effects of cavitation number on the water entry process of supercavity blade section. Based on the force plots, it can be inferred that Cavitation number or better yet the ambient pressure may not significantly affect the forces in both fully ventilation and partially cavitation conditions. It is evident that magnitudes of the forces are approximately equal for both Cavitation numbers. However, for the transition condition, it is observed that the case with larger Cavitation number/ambient pressure have larger forces. This may be attributed to the intensive vaporization in the cavity area of the case with smaller cavitation number, which was explained earlier. It is also evident that transition condition of the case with smaller Cavitation number exhibits a delay, in comparison with the other case. Finally, the plots of the efficiency show that there is no remarkable change in efficiency for both of the considered cases. On the other hand, it can be stated that Cavitation number may not significantly affect the efficiency for the investigated velocity ratios. This may be attributed to the reduction of both horizontal and vertical forces in the case of smaller Cavitation number.
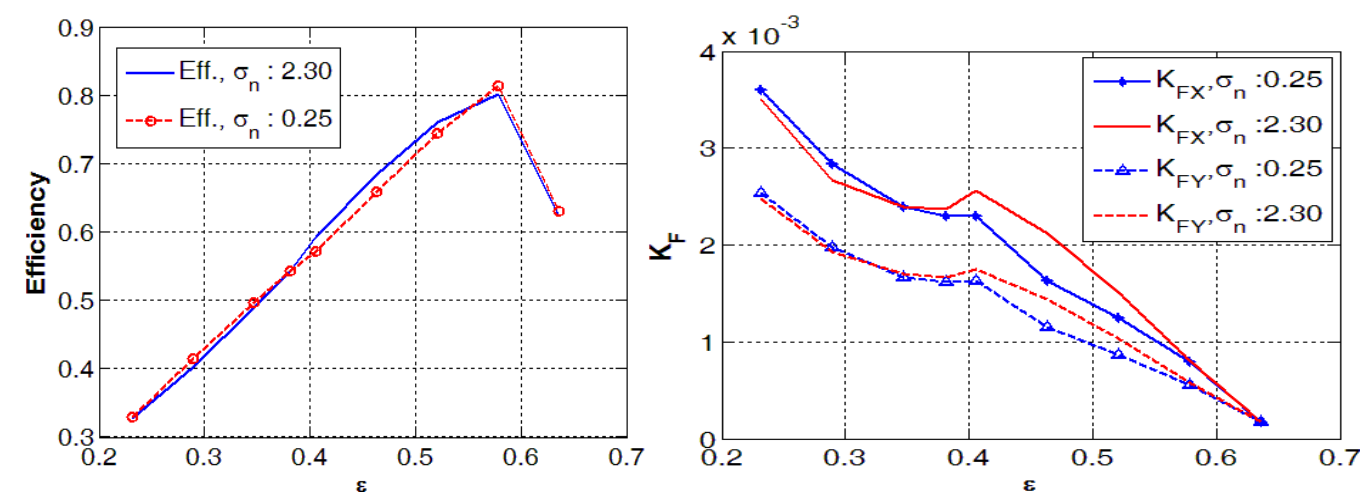

Fig. 20. Forces (right) and efficiency (left) of the foil for two different cavitation numbers.

\section{Conclusions}

In the current paper, numerical simulation of flow around a thin wedge is presented in order to provide a better understanding of the effects of Cavitation number and advance ratio/velocity ratio on the performance of surface piercing propellers in different conditions. Coupled URANS equation, VOF scheme, and Zwart's cavitation model [33] are utilized to conduct the targeted numerical simulations. 
Probing into the effects of cavitation on hydrodynamic characteristics of surface piercing propellers through numerical modeling of oblique water entry of a thin wedge

The validity of the proposed model is assessed in two steps. First, the water entry of a wedge is modeled and the obtained results indicate good accuracy for the computed pressure and free surface elevation. Subsequently, cavitation phenomenon around a supercavitating hydrofoil is modeled. It is demonstrated that cavity length, pressure, and lift force are predicted with good accuracy.

The proposed model is then used to investigate the defined problem for two different Cavitation numbers. The produced results demonstrate that in fully ventilated condition, smaller ambient pressure may not affect the pressure distribution as well as the free surface profile. However, in the transition condition, it is observed that the case with smaller Cavitation number has lower pressure difference on the wedge's sides, since vapor is produced. Also, the results of horizontal and vertical forces in this regime reveal that these forces reduce, as the ambient pressure decreases. It should also be noted that as the Cavitation number decreases, the transition mode appears at a larger velocity ratio. The results of efficiency coefficient indicate that a decrease in the Cavitation number may not affect this coefficient for the investigated range of velocity ratio.

Future studies may include numerical modeling of the fluid-structure interaction for these sections by considering both one-way and two-way coupling. Numerical modeling of the problem in three-dimensional condition may be considered another future task.

\section{Acknowledgment}

This research received no specific grant from any funding agency in the public, commercial, or not-for-profit sectors and there is no conflict of interest.

\section{References}

[1] Shiba, H., (1953). Air-drawing of marine propellers. Technical Report 9, Transportation, Technical Research Institute.

[2] Hadler, J.B., Hecker, R., (1968). Performance of Partially Submerged Propellers. Proc 7th ONR Symposium on Naval Hydrodynamics, Rome.

[3] Hecker, R., (1973). Experimental Performance of a Partially Submerged Propeller in Inclined Flow. SNAME Spring Mtg; Lake Buena Vista, Florida.

[4] Kruppa, CFL. (1972). Testing of Partially Submerged Propellers. Proc 13 ITTC Report of Cavitation, Berlin \& Hamburg, Appendix V, y 1.

[5] Brandt, H. (1973). Modellversuche mit Schiffspropellern an der Wasseroberfiache. Dissertation TU Berlin,.

[6] Olofsson, N., (1993). A Contribution on the Performance of Partially Submerged Propellers. Proc FAST '93, Yokohama, Japan, V1, pp 765-776.

[7] Shen, Y.T., (1974). General Scaling Problems on Fully Cavitating and Ventilated Flows, Proceedings of the 17th ATTC, Pasadena.

[8] Furuya, O., (1985). A performance-prediction theory for partially submerged ventilated propellers, J. Fluid Mech., vol. 151, pp. 311-335. https://doi.org/10.1017/S0022112085000982.

[9] Wang, G., Zhu, X., Sheng, Z., (1990). Hydrodynamic forces of a three dimensional fully ventilated foil entering water. Journal of Hydrodynamics, 5(2). 

characteristics of surface piercing propellers through numerical modeling of oblique water entry of a thin wedge

[10] Kudo, T., Kinnas, S., (1995). Application of vortex/source lattice method on supercavitating propellers. In 24th American Towing Tank Conference, College Station, TX.

[11] Young, Y.L., Kinnas, S.A., (2003). Analysis of supercavitating and surface-piercing propeller flows via BEM, Computational Mechanics 32. https://doi.org/10.1007/s00466-003$\underline{0484-6}$

[12] Young, Y.L, Savander, B.R., (2011). Numerical analysis of large-scale surface-piercing propellers, Ocean Engineering. https://doi.org/10.1016/j.oceaneng.2011.05.019.

[13] Ghassabzadeh, M., Ghassemi, H., Saryazdi, M.(2013). Determination of Hydrodynamics Characteristics of Marine Propeller Using Hydro-elastic Analysis. Brodogradnja, 64(1),4045 .

[14] Ekinci, S., Celik, F.,Guner, M. (2010). A Practical Noise Prediction Method for Cavitating Marine Propellers, Brodogradnja, 61(4), 359-366.

[15] Bagheri, M.R., Mehdigholi, H., Seif, M.S., Yaakob, O., (2015). An experimental and numerical prediction of marine propeller noise under cavitating and non-cavitating conditions, Brodogradnja, 66(2),29-45.

[16] Kinaci, O.K., Gokce, M.K. (2015). A computational hydrodynamic analysis of Duisburg test case with free surface and propeller. Brodogradnja, 66(4), 23-38.

[17] Samir, E.B., Mohammed, A., Omar, I., Fahri, C.(2016). Effect of geometric configurations on hydrodynamic performance assessment of a marine propeller, Brodogradnja, 67(4), 31-48.

[18] Yim, B., (1974). Linear theory on water entry and exit problems of a ventilating thin wedge. J. Ship Res. 18 (1), 1-11.

[19] Wang, D.P., (1977). Water entry and exit of a fully ventilated foil. J. Ship Res. 21 (1), 44-68. https://doi.org/10.21236/ADA065007.

[20] Cox, B.D. (1971). Hydrofoil theory for vertical water entry. PhD thesis, Massachusetts Institute of Technology, Cambridge, MA.

[21] Judge, C., Troesch, A., Perlin, M., (2004). Initial water impact of a wedge at vertical and oblique angles. J. Engineering Math, 48(3), 279-303.

[22] Faltinsen, O.M., Semenov Y.A. (2008). Nonlinear problem of flat-plate entry into an incompressible liquid. J. Fluid Mech. 611, 151-173. https://doi.org/10.1017/S0022112008002735.

[23] Xu, G.D., Duan, W.Y., (2008). Numerical simulation of oblique water entry of an asymmetrical wedge, Ocean Engineering, 35, 1597-1603. https://doi.org/10.1016/j.oceaneng.2008.08.002.

[24] Xu, G.D., Duan, W.Y., Wu, G.X., (2010). Simulation of water entry of a wedge through free fall in three degrees of freedom. Proceedings of the Royal Society, 486, 2219-2239.

[25] Ghadimi, P., Saadatkhah, A., Dashtimanesh, A., (2011). Analytical solution of wedge water entry by using schwartz-christoffel conformal mapping. Int J Modeling, Simulation and Scientific Computing. 2(3): 337-354.

[26] Ghadimi, P., Dashtimanesh, A., Djeddi, S.R., (2012). Study of water entry of circular cylinder by using analytical and numerical solutions. J Brazilian Society of Mech Sci Eng. 37(3), 821-835.

[27] Ghadimi, P., Feizi Chekab, M.A., Dashtimanesh, A. A., (2013). Numerical Investigation 
Probing into the effects of cavitation on hydrodynamic characteristics of surface piercing propellers through numerical modeling of oblique water entry of a thin wedge

of the Water Impact of an Arbitrary Bow Section, ISH Journal of Hydraulic Engineering, 19(3): 186-195. http://dx.doi.org/10.1080/09715010.2013.796690.

[28] Ghadimi, P., Feizi Chekab, M.A., Dashtimanesh, A. (2014). Numerical simulation of water entry of different arbitrary bow sections. J Naval Archit Marine Eng. 11 (2): 117-129. https://doi.org/10.3329/jname.v11i2.18724.

[29] Farsi, M., Ghadimi, P., (2014a). Finding the best combination of numerical schemes for 2D SPH simulation of wedge water entry for a wide range of deadrise angles. Int $J$ Naval Archit Ocean Eng. 6: 638-651. https://doi.org/10.2478/IJNAOE-2013-0202.

[30] Farsi, M., Ghadimi, P., (2014b). Effect of flat deck on catamaran water entry through smoothed particle hydrodynamics. Institution of Mechanical Engineering Part $M: J$ Engineering for the Maritime Environment, Published Online: March.

[31]Farsi, M., Ghadimi, P., (2015). Simulation of 2D symmetry and asymmetry wedge water entry by smoothed particle hydrodynamics method. J Brazilian Society of Mech Sci Eng. 37(3), 821-835. https://doi.org/10.1007/s40430-014-0212-5.

[32] Feizi Chekab, M.A., Ghadimi, P., Farsi, M., (2015). Investigation of threedimensionality effects on aspect ratio on water impact of 3D objects using smoothed particle hydrodynamics method. J Brazilian Soc Mech Sci Eng. Published Online: June. 2015. DOI: 10.1007/s40430-015-0367-8. https://doi.org/10.1007/s40430-015-0367-8.

[33] Zwart P, Gerber, AG. \& Belamri, T. A. (2004). Two-Phase Model for Predicting Cavitation Dynamics. ICMF International Conference on Multiphase Flow, Yokohama, Japan.

[34] Ji, B., Luo, X.W., Wang, X., Peng, X.X., Wu, Y.L., Xu, H.Y., (2011). Unsteady numerical simulation of cavitating turbulent flow around a highly skewed model marine propeller. J. Fluids Eng.-Trans. ASME, 133, 011102. https://doi.org/10.1115/1.4003355.

[35] Mejri, I., Bakir, F., Rey, R., Belamri, T., (2006). Comparison of computational results obtained from a homogeneous cavitation model with experimental investigations of three inducers. J. Fluids Eng.-Trans. ASME 128, 1308-1323. https://doi.org/10.1115/1.2353265.

[36] Tveitnes, T., Fairlie-Clarke, A.C., Varyani, K.,(2008). An experimental investigation into the constant velocity water entry of wedge-shaped sections. J. Ocean Engineering 35, 14631478. https://doi.org/10.1016/j.oceaneng.2008.06.012.

[37] Dinh, N.N. (1968). Some Experiments on a Supercavitating Plane Hydrofoil with JetFlap, SNAME.

[38] Wu, T.Y., (1956). A Free-Streamline Theory for Two-Dimensional Fully Cavitated Hydrofoils, Journal of Mathematics and Physics, vol. 35, Issue 1-4, pages 236-265, https://doi.org/10.1002/sapm1956351236

Submitted: 22.02.2017. Nasrin Javanmardi, n.javan@aut.ac.ir

Dept. of Marine Technology, Amirkabir University of Technology, Tehran, Iran

Parviz Ghadimi, pghadimi@aut.ac.ir

Dept. of Marine Technology, Amirkabir University of Technology, Tehran, Iran

Sasan Tavakoli, sasan_tavakoli@aut.ac.ir

Dept. of Marine Technology, Amirkabir University of Technology, Tehran, Iran 